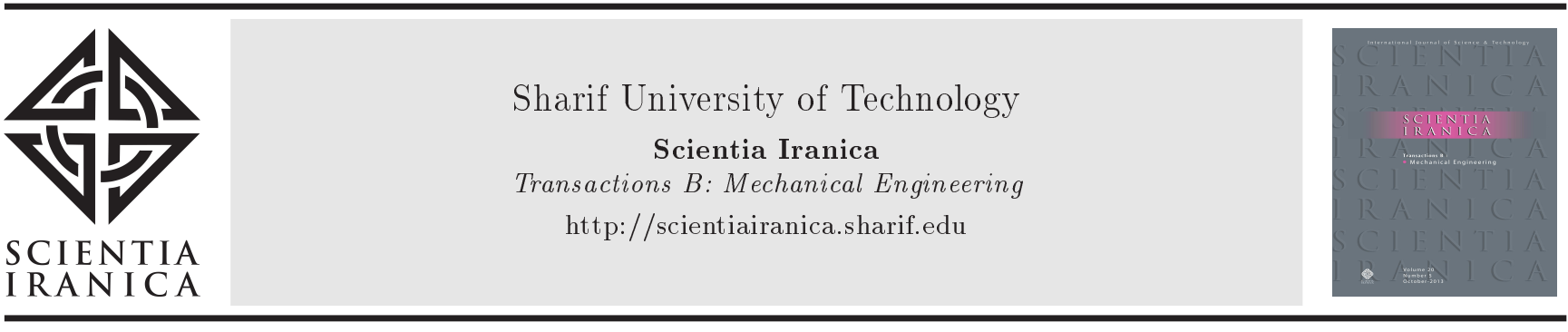

\title{
Molecular dynamics simulations of sessile and pendant droplets' shape on inclined and curved surfaces
}

\author{
M.J. Javanmardi ${ }^{a}$, K. Hirbodi ${ }^{a}$, A. Avara ${ }^{a}$, and M. Yaghoubi $i^{a, b, *}$ \\ a. School of Mechanical Engineering, Shiraz University, Shiraz, P.O. Box 71936-16548, Iran. \\ b. The Academy of Sciences of IR Iran, Tehran, P.O. Box 19735-167, Iran. \\ Received 19 February 2018; received in revised form 19 June 2018; accepted 11 August 2018
}

\author{
KEYWORDS \\ Molecular dynamics; \\ Contact angle; \\ Hysteresis; \\ Inclined plate; \\ Curved surface; \\ Sessile drop; \\ Pendant drop.
}

\begin{abstract}
In this study, shapes of water droplets with different sizes on various inclined smooth surfaces are simulated numerically, and advancing and receding contact angles are determined using the molecular dynamics approach. Experimental measurements are also carried out to validate the numerical predictions of droplets' shape on inclined surfaces. Based on the verified code, shapes of water droplets with different sizes around smooth circular cylinders with various diameters are simulated. Furthermore, advancing and receding contact angles along with the hysteresis values of the sessile and pendant droplets with various sizes around the cylinders are evaluated. Finally, based on the numerical results, two correlations are developed to predict advancing and receding contact angles of droplets on the circular cylinders. According to the results, maximum advancing and minimum receding angles take place on both sides of the cylinder on a horizontal line passing through the cylinder center. As a result, contact angle hysteresis reaches its maximum value in these two positions. In addition, advancing and receding angles have the same values on the top and bottom of the cylinder. Moreover, droplet size and cylinder diameter have minor effect, while drop position has major effect on the shape of droplets over the cylinder. (C) 2018 Sharif University of Technology. All rights reserved.
\end{abstract}

\section{Introduction}

Heat transfer coefficient in drop-wise condensation is much higher than that in film-wise condensation [1]. This overwhelming aspect of drop-wise condensation has made it an outstanding candidate for researchers to conduct widespread studies on its basic physical aspect as well as on its various applications. Numerous experimental and numerical studies have been made so far on this attractive field of engineering interest. Abu-Orabi [2] developed a model using the population

\footnotetext{
*. Corresponding author. Tel./Fax: +98 7132301672 E-mail addresses: mjavan@shirazu.ac.ir (M.J. Javanmardi); kamran_hirbodi@shirazu.ac.ir (K. Hirbodi); avara@pgu.ac.ir (A. Avara); yaghoubi@shirazu.ac.ir (M. Yaghoubi)
}

doi: $10.24200 /$ sci. 2018.20832 balance concept to predict drop size distribution for small drops that grow through direct condensation. Wu et al. [3] applied the random fractal model to simulate the drop size and special distribution in drop-wise condensation. Vemuri and Kim [4] created hydrophobic coatings on copper alloy surfaces to enhance steam condensation through drop-wise condensation. In their work, similar to the work of Abu-Orabi [2], a model based on the population balance concept was used to derive a theoretical formula to predict the drop size distribution of small drops that grow through condensation. Rausch et al. [5] achieved a stable drop-wise condensation of saturated steam on an aluminum alloy disc by means of ion beam implementation technology. Mei et al. [6] developed a fractal model for drop-wise condensation heat transfer based on the fractal characteristics of drop size distributions on condensing surfaces. Do Hong et al. [7] predicted static 
and dynamics contact angles of water droplets on a solid surface using the molecular dynamics method. They realized that the increase of characteristic energy of the solid surface led to a decrease in the static contact angle due to changing the hydrophobic surface to a hydrophilic one. Rausch et al. [8] achieved stable drop-wise condensation of steam by ion beam implementation of $\mathrm{N}^{+}$on titanium surfaces stabilized by a peroxidation procedure. Lara and Holtzapple [9] measured heat transfer coefficient in vertical dimpledsheet heat exchangers, some of which were bare and the others were coated with a thin layer of passive electroless Ni-P-PTFE. In addition, Lara and Holtzapple [10] carried out an experimental investigation of hydrophobic heat exchangers. Two plates were studied; one was bare, while the other had a hydrophobic coating. Moreover, Mei et al. [11] simulated processes of nucleation, growth, re-nucleation, and sweeping of droplets based on the intrinsic growth rate of a single droplet. Their studies were related to influences of the number of initial droplets on the average radius, surface coverage, and number of droplets on substrate. Their results showed that the apparent growth rate of droplets was strongly dependent on the number of initial droplets. Guilizzoni [12] experimentally investigated the shape and contact angle of drops on curved surfaces. They used image processing, spline fitting, and numerical integration to extract the drop contour in a number of cross sections. Park et al. [13] investigated numerically some wetting characteristics, such as contact angle and wetting radius of water drops, on solid surfaces with various roughness states. The molecular dynamics simulation was implemented to analyze the wetting behavior of water droplets on the surfaces. They figured out that contact angles for a hydrophobic surface with low characteristic energy were rarely dependent on the roughness height. However, the contact angles for surfaces with high characteristic energy were dependent on both the roughness height and droplet size. Sergi et al. [14] used the molecular dynamics approach to determine the contact angle of water droplets with an initial radius ranging from 20 to $80 \AA$ spreading on graphite surfaces. They found that, for small droplets, surface thickness fluctuations are very effective in deforming their spherical shape. Bonner [15] presented two generalized correlations for drop-wise condensation heat transfer coefficient: heat flux dependence and non-heat flux dependence. Eral et al. [16] reviewed those studies on the contact angle hysteresis. They discussed the most important theoretical, experimental, and numerical studies in their paper. Based on the numerical investigations, the continuum approach, such as lubrication theory, and the molecular approach, such as molecular dynamics simulation, are two different approaches to analyze contact angles. Santiso et al. [17] calculated the solid-fluid contact angles using the molecular dynamics simulations. Their approach was able to approve that surface roughness and energetic heterogeneities affected the contact angle. Wu et al. [18] proposed a theoretical model to estimate the contact angle of droplets on convex and concave surfaces. This model mainly depends on droplet volume, surface curvature, and the contact angle of the droplet on a flat surface. Kim et al. [19] measured the dynamics advancing and receding contact angles on hydrophobic and super-hydrophobic surfaces as a function of plate velocity and capillary number. They found that the advancing contact angle remained constant as independent of capillary number, while the dynamics receding contact angle decreased with increasing capillary number. Cheng et al. [20] proposed a mathematical model to determine the contact angle hysteresis by employing concepts of surface-energy minimization. The model can predict contact angle hysteresis of droplets on different surface geometries and degrees of wetting. Results show that contact angle hysteresis increases with increasing the surface roughness and degree of wetting. Burt et al. [21] studied the effect of drop size and surface interaction on the contact angle of ionic-liquid droplets by molecular dynamics simulations. Their study revealed that there was no change in contact angle with drop size at low interaction potential. However, the contact angle strongly reduces when the surface interaction potential increases. Contact angle and wetting behavior of soil minerals were investigated by Zhang et al. [22]. The molecular dynamics simulation was employed, and three types of the soil minerals, including a-quartz, orthoclase, and muscovite, were considered. This study indicated that the contact angles intensively were dependent on the soil mineral types. Wu and Ma [23] proposed a new model based on the fractal structure of the contact line to make a connection between three different contact angles including the equilibrium, receding and advancing contact angles. This model has only one parameter and can be easily reduced to other models proposed by prior investigators. Škvára et al. [24] proposed a new method from molecular simulations to evaluate the contact angle of droplets on a solid underlay. The accuracy of this method strongly depends on the droplet size. For very small droplets, determination of contact angle is complicated, while larger droplets do not significantly affect the contact angle. Villa et al. [25] presented two theoretical models to predict the effect of surface temperature on the contact angle. They validated the new models with experimental measurements. Based on their results, the contact angle decreases with increasing surface temperatures on the hydrophilic surface, whereas the contact angle remains almost constant on hydrophobic surfaces for increasing wall temperatures. Feng et al. [26] studied the impact of temperature and particle 
size on the contact angle of $\mathrm{Cu} / \mathrm{Si}\left(\begin{array}{lll}1 & 1 & 1\end{array}\right)$ system by the molecular dynamics approach. They observed that contact angle decreased with increasing temperature and decreasing particle size of the system.

Literature includes many works that have been conducted theoretically, numerically, and experimentally to simulate drop-wise condensation, a few of which have been reviewed in the previous paragraph. However, new numerical simulations, modeling, theories, approaches, and new applications of this interesting phenomenon are still under consideration for many researchers. Drops nucleation, formation, growth, coalescence, departure, sweeping, dripping, and regeneration on a surface, in addition to the drops shape on inclined surfaces, are all chaotic phenomena occurring in various engineering system configurations. This stimulus is sufficient for researchers to continue their theoretical and experimental studies on further understanding and developing of practical approaches to enhance convection heat transfer considering dropwise condensation. For such an aim, the present motivation is to apply molecular dynamics theory as a new scheme to develop a certain aspect of drop formation on the surfaces.

In this paper, an in-house computational code is developed to predict the shape of water droplets formed on inclined and curved surfaces with the implementation of the molecular dynamics method. Sizes of droplets are selected as 5, 10, and $15 \mu \mathrm{L}$, and inclination angles of the plate are considered as $0,45,60$, and $90^{\circ}$ relative to the horizontal axis. Furthermore, circular cylinders with diameters of 25 , 35 , and $70 \mathrm{~mm}$ are considered as the curved surface. In order to validate the numerical approach, outputs of the simulations for the shape of droplets are compared with the experimental measurements on inclined surfaces. Finally, the verified code is employed to predict the advancing and receding contact angles of sessile and pendant water droplets on the circular cylinders. Moreover, based on the determined angles, contact angle hysteresis is calculated, and two correlations for estimating advancing and receding contact angles are proposed. Details of the numerical modeling are introduced in the following section. The experimental observations are explained in the next section; finally, the obtained results are compared and discussed.

\section{Methodology}

The general aspects of the molecular dynamics approach for predicting the shape of drops formed on surfaces are briefly introduced in this section. More details of this method are provided in the appendix.

In this approach, a water drop is assumed to be made up of a number of small spherical particles. Each particle consists of several actual molecules. The adequate number of molecules is determined based on interaction forces between a pair of particles so that they become independent of the number of particles. The particles constituting the drop can slide over each other due to various forces acting on them. In general, the forces applied to each particle may be the gravitational force of the earth as well as the cohesive forces of the other same particles in its neighborhood. Moreover, for those particles in the vicinity of a solid surface, the adhesive force of the surface is included, too. Note that the cohesive forces are the intermolecular forces that exist between molecules of the same substance, while the adhesive forces are attractive forces between unlike molecules. The most important methods to determine these two molecular interactions were reviewed by Rosenholm et al. [27].

However, Newton's second law of motion is applied to track each particle in the drop. Once the position of all particles is known for each time step, the shape of the drop can be consequently determined. It should be noted that the foregoing procedure should continue until the changes in the predicted shape of the drop become negligible. This means that the drop has approached its equilibrium condition on the specified surface.

The procedure begins with assuming an arbitrary initial shape for the boundary of the water drop. Then, the particles are distributed in its boundary. The number of particles is expected to be large enough so that they, as a whole, could well resemble the drop shape. Given the number of the particles, their size is also known since their volumes should essentially sum up the volume of the drop.

Once the particles are given an initial distribution in the drop, the forces acting on each particle are to be determined. As already stated, there are various forces that act on each particle. These forces are the particle's weight, the cohesive forces due to the neighboring particles, and the adhesive force originating from the solid surface. The adhesive force of the solid surface, however, is necessary to consider only for those particles in its neighborhood. In order to determine the cohesive forces acting on a specific particle, its neighbors are to be identified. Consequently, a search through all the other particles has to be made to find the neighbors of that specific particle. The searching algorithm is repeated for all particles in each time step. Therefore, this part of the program is rather time consuming. Figure 1(a) illustrates an initial distribution provided for the particles near a horizontal solid surface. Note that the arrangement given to the particles is arbitrary. A fraction of the particles is also shown in Figure 1(b). The position of a specific particle, such as particle $i$, in the middle of its neighbors and the forces acting on it are also shown in Figure 1(b). 


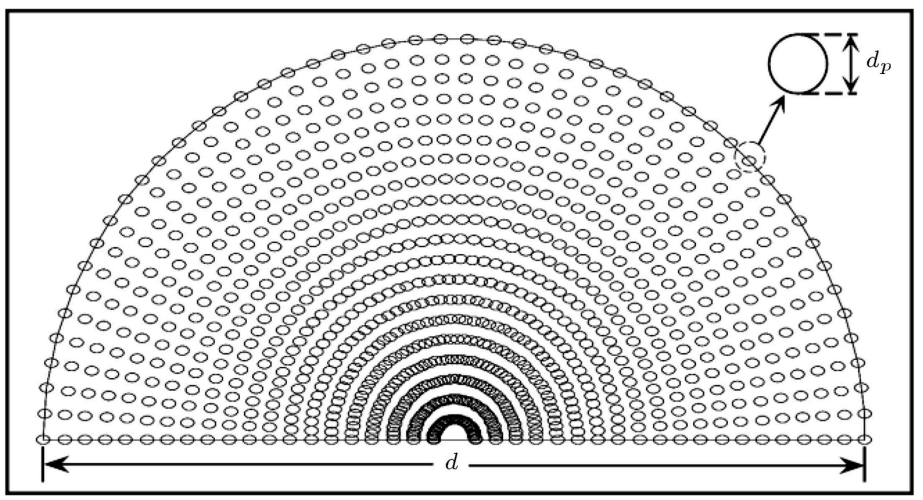

(a)

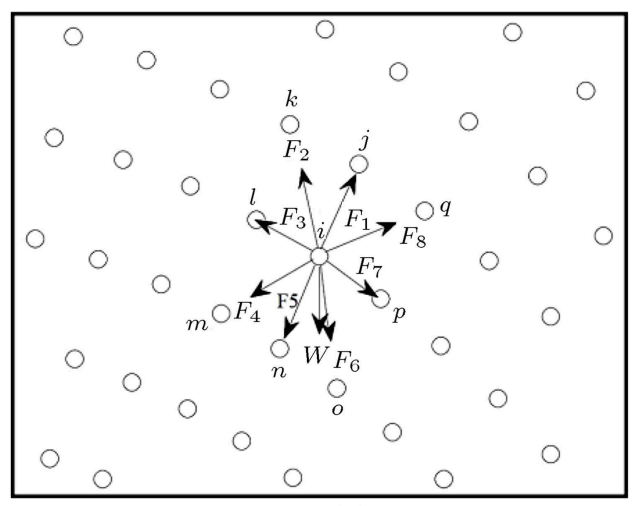

(b)

Figure 1. (a) Schematic of an initial distribution of particles in a water drop. (b) Forces acting on a particle.

Every particle in this drop can move through other particles, and Newton's second law governs the motion of each particle. This equation is written for particle $i$, shown in Figure 1(b), as follows:

$$
\vec{F}_{i}=m \vec{a}_{i}
$$

In Eq. (1), $\vec{F}_{i}$ is the summation of all forces acting on particle $i$, and $\vec{a}_{i}$ is the particle acceleration vector. Subscript $i$ is not given to $m$, since mass is assumed the same for all the particles in the drop. Hence, the equation of motion should also be written for all the particles.

Writing the equation of motion for each particle returns its acceleration vector to the beginning phase of a time step. The integration of this equation with respect to time gives the velocity vector of the particle, and a subsequent integration determines the position vector of the particle at the end of the time step. If one chooses the leapfrog method well described in [28] to carry out the numerical integrations of the equation of motion, velocity and position vectors will be respectively computed as follows:

$$
\begin{aligned}
& \vec{u}_{i}(t+\Delta t / 2)=\vec{u}_{i}(t)+(\Delta t / 2) \vec{a}_{i}(t), \\
& \vec{r}_{i}(t+\Delta t)=\vec{r}_{i}(t)+\Delta t \vec{u}_{i}(t+\Delta t / 2), \\
& \vec{u}_{i}(t+\Delta t)=\vec{u}_{i}(t+\Delta t / 2)+(\Delta t / 2) \vec{a}_{i}(t+\Delta t) .
\end{aligned}
$$

In these equations, $\Delta t$ represents the time step. In addition, Eq. (2) determines the velocity vector of the particle in the middle of the time step. Using the data obtained from this equation, the position vector of the particle at the end of the time step can be subsequently evaluated by Eq. (3). Finally, the implementation of Eq. (4) gives the velocity vector of the particle at the end of the time step.

The particles that move near the surface are assumed to undergo conservative impact with the surface,

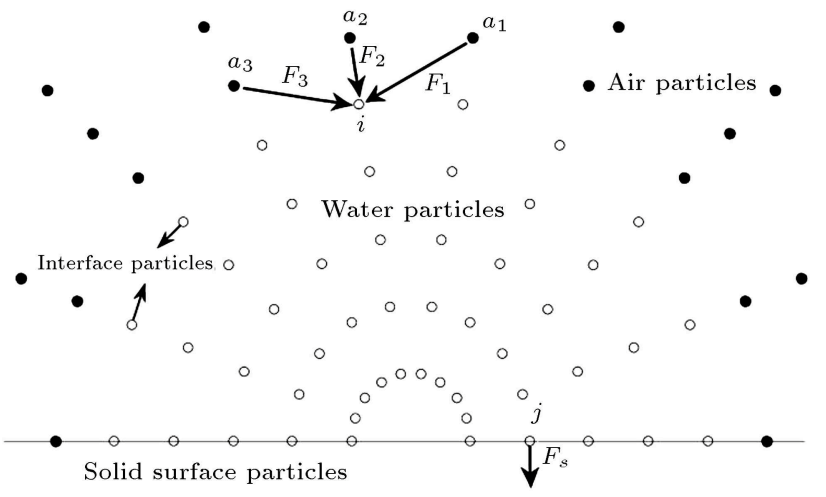

Figure 2. Schematic of drop particles surrounded by surface and air particles, forces acting on air particles $a_{1}$, $a_{2}$, and $a_{3}$ on particle $i$ of the drop, and the surface force acting on particle $j$ of the drop.

while the adhesive force of the surface is applied to them. On the other hand, the particles moving at the outer boundary of the droplet are assumed to be influenced by the force resulting from the air pressure upon them. For such consideration, virtual air particles of the same size as water particles of the drop are thus considered to exist at the outer boundary of the droplet. In addition, the gravitational force of these virtual air particles is then considered as the air force acting on the water particles, as illustrated in Figure 2.

Once the position vectors of the particles are known, an approximated shape can be drawn for the drop. This shape will essentially differ from the one imagined for the drop as an initial guess. It may also differ, however, from the correct shape of the drop. In order to approach the correct prediction for shape of the drop, computations should be continued for the next time step. The position and velocity vectors already computed are to be used for the calculations required in the next time step. This procedure goes on until the final shape of the drop is determined, and further calculations do not significantly change the drop shape. 
In this paper, the foregoing methodology is implemented to predict the shape of a water droplet formed on an inclined surface. The surface material is assumed smooth, and its inclination angle is given variable values. Size of the droplets is also taken as a variable. For each case, the drop shape and its contact angles are determined. In addition, experimental measurements are conducted in parallel to the numerical calculations to visualize the real drop shape for various surface inclinations. Using the photos taken from the experiment, the real contact angles of the droplets are then measured. Furthermore, a comparison is made between the calculated and measured contact angles to validate numerical results. Finally, the validated numerical method is applied to predict the shape of sessile and pendant drops with various sizes on circular cylinders with different diameters.

\section{Experimental setup}

To validate numerical computations and calibrate the contact angle, some experiments were conducted to measure the drop contact angles on inclined surfaces. The liquid used is water, and the surfaces on which drops are placed are super-hydrophobic aluminum, made by using etching and stearic acid monolayer assembling as recommended by $\mathrm{Wu}$ et al. [29]. The process of making a super-hydrophobic aluminum can be stated in the following steps in brief:

1. The cleaned aluminum 7000 group alloy piece was first polished with granularities of $600 \#, 800 \#$, and $1200 \#$ as well as metallographic sanding papers gradually for about 1 hour. The oxide layers of aluminum alloy surface were removed during this process;

2. After ultrasonically cleaning the sample in ethanol and de-ionized water for about 15 minutes, it was dried at room temperature, followed by immersing in the acidic solution for 4 hours. The acidic solution was an aqueous solution of 1 mole/L (3.7\%) hydrochloric acid. The sample after immersing in the above solution was washed with de-ion water and dried;

3. Dilute potassium permanganate $\left(25^{\circ} \mathrm{C}, 0.1 \mathrm{~mole} / \mathrm{L}\right)$ was employed to obtain a more stable surface; the immersing time in $\mathrm{KMnO}_{4}$ was about 1 hour;

4. Finally, the sample was immersed into $1 \%$ stearic acid for about 30 minutes to further reduce surface energy.

In addition, the procedure of measurements was done in the following steps:

1. The tilted plate is adjusted at the required angle;

2. The microscope and attached camera are placed as close to the drop, approximately $1 \mathrm{~cm}$ length;

3. The water drop is released with micro-injector on the prepared hydrophobic surface when it is horizontal;

4. The aluminum surface is placed on the titled plate;

5. The camera is zoomed to see the drop shape clearly;

6. The pictures are taken within 30 seconds when room temperature is about $25^{\circ} \mathrm{C}$.

Drops are injected onto the test surface by a microsyringe when the surface is horizontal. If the surface tiltes immediately, the drop will roll and fall from the surface. Then, the surface tilts after 3 minutes to the required inclination angle. The maximum uncertainty in the volume measured using a syringe is $2.5 \%$. In order to prevent evaporation from the drop surface, the whole setup is placed in a box, in which air is saturated at room temperature, and the box is placed on a firm horizontally leveled table.

Profile images of droplets are taken by using a Dino-Lite Premier AM4113T microscope and an attached camera, as shown in Figure 3. The images are recorded in a $\mathrm{PC}$ and used to measure contact angles using image-processing software. At least four pictures were recorded for each angle so that average values of

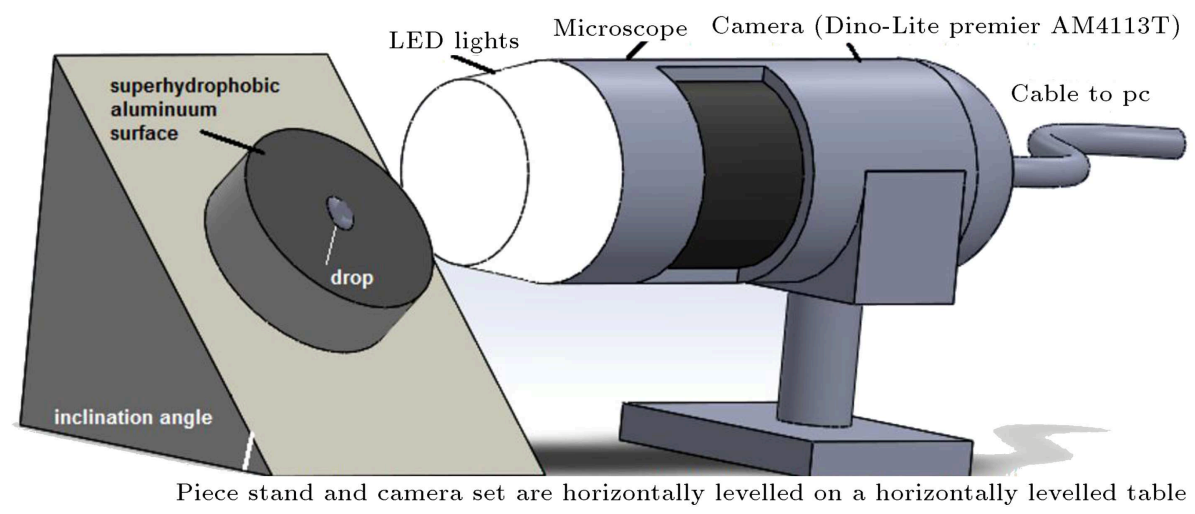

Figure 3. Schematic of the experimental arrangement to measure drop contact angles. 
contact angles were obtained. The maximum deviation of the measured angles from the average angle was $1.5^{\circ}$.

\section{Results and discussion}

In this study, water droplets of 5,10 , and $15 \mu \mathrm{L}$ are examined. The surface on which drops are formed is assumed to be smooth and hydrophobic with inclination angles of $0,45,60$, and $90^{\circ}$ with respect to the horizontal direction. For each case, the shape of the water drop is predicted using the presented methodology, and both of its advancing and receding contact angles are calculated.

More than 10,000 particles with $0.1 \mathrm{~mm}$ diameter are distributed in the drop. This number of particles is large enough to simulate a drop shape. Moreover, with the value given to the diameter of the particles, their mass is thus equal to $5.236 \times 10^{-10} \mathrm{~kg}$, which is the same for all the particles. In addition, time step is given the value of $10^{-5} \mathrm{~s}$ in this work, which is sufficiently small to consider minor displacements of the particles.

Evaluation of the cohesive forces acting among the particles is conducted using molecular dynamics theory [28]. Description of this scheme is explained in the Appendix. The present technique started with an assumed value given to the adhesive force of the surface. With this assumption, a drop formed on a horizontal surface is modeled first. Considering the value of the drop contact angle known from the experimental measurements, a trial and error procedure is used for evaluating the value of the adhesive force of the surface until the drop contact angle obtained from the numerical technique becomes equal to that obtained from the experimental measurements. The resulting magnitude for the adhesive force is then used as constant during the rest of the calculations for the other drop sizes and surface orientations. The numerical approach is repeated to model the shape of the water droplets over inclined surfaces, and their advancing and receding contact angles are compared with the experimental measurements. However, since the experimental measurements are carried out for smooth hydrophobic surfaces, the present numerical calculations are valid only for surfaces with smooth and hydrophobic aluminum characteristics. Finally, the numerical technique is employed to predict the shape of the drops formed around circular cylinders with diameters of 25,35 , and $70 \mathrm{~mm}$ to generalize the computations.

Figure 4 shows the initial shape assumed for the water drop of volume $5 \mu \mathrm{L}$, which is to be formed on a horizontal surface. Since the procedure continues until the final shape for the drop is reached, this shape seems convenient as an initial guess.

At the end of each time step, when the new position vectors of all particles are calculated, a curve

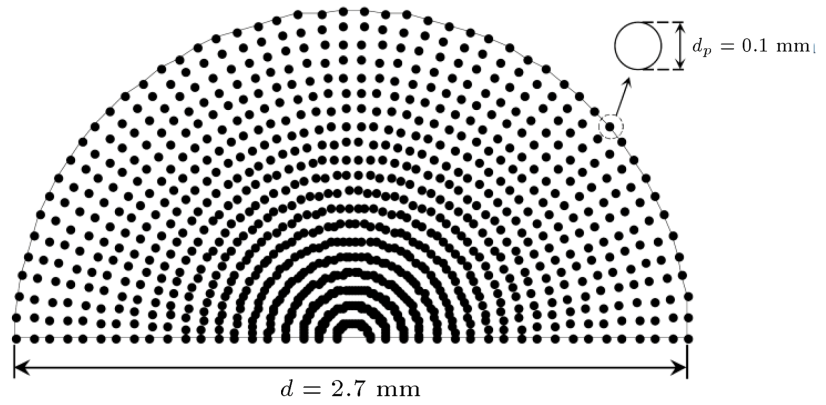

Figure 4. Initial distribution of particles in a drop with 5 $\mu \mathrm{L}$ volume, drop diameter of $2.7 \mathrm{~mm}$, particle diameter of $0.1 \mathrm{~mm}$, and number of particles of 10,000 .

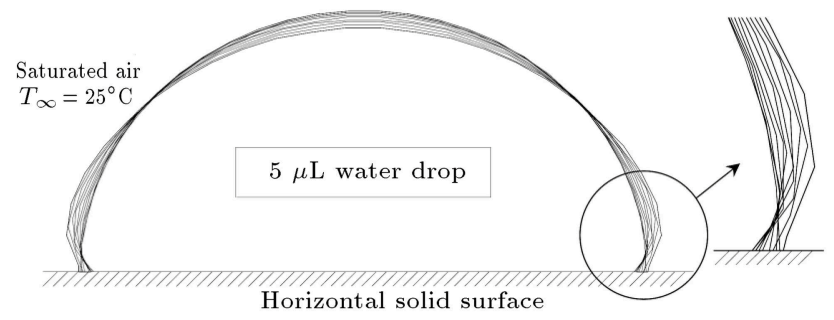

Figure 5. Drop shape predicted by the computational code during several time steps.

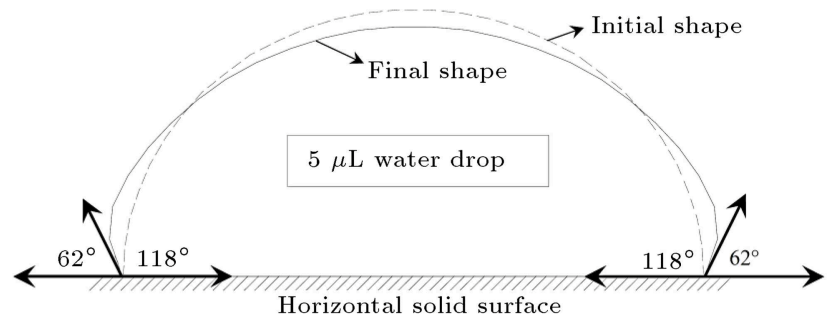

Figure 6. Initial and final shapes of a drop formed on a horizontal hydrophobic surface.

that encloses all the particles is drawn. This curve shows the shape approximated for the drop at the end of the respective time step. The program goes on until this curve does not change considerably with time. A few of these curves for the case of horizontal surface at different times are illustrated in Figure 5. This figure also shows how the drop contact angle changes toward its final value during successive time steps.

Although Figure 5 includes some of the contours successively drawn for the drop shape, Figure 6 is also given to make a better comparison of the initial shape with $90^{\circ}$ contact angle and final shape of the drop with the consequent contact angle. According to this figure, the volume of the drop remains constant during the evolution of its shape in the final state.

The image taken from the experimental measurements of the real shape for a $5 \mu \mathrm{L}$ drop on a horizontal smooth hydrophobic surface is given in Figure 7(a). The measured contact angles, which are equal to $115.8^{\circ}$ for the right and $112.7^{\circ}$ for the left, are also given in 


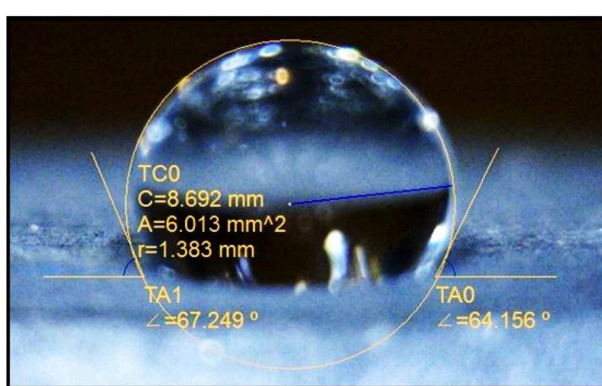

(a)

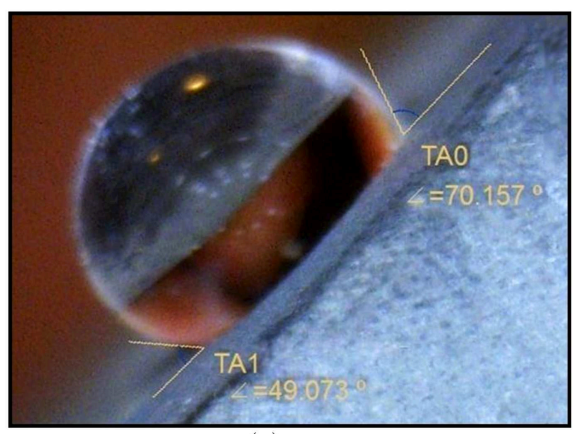

(c)

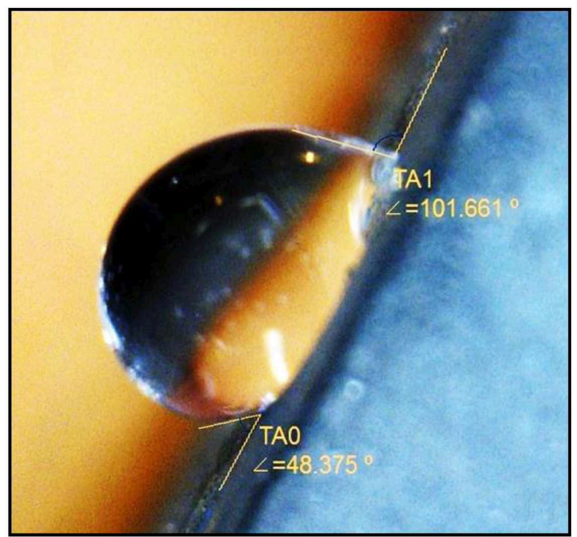

(e)

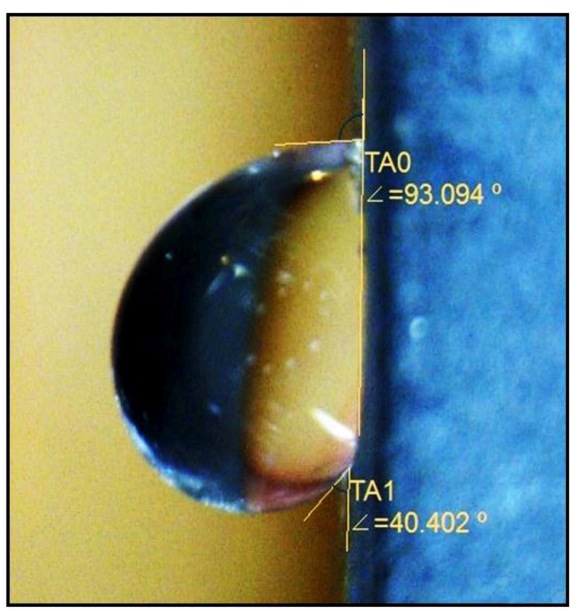

$(\mathrm{g})$

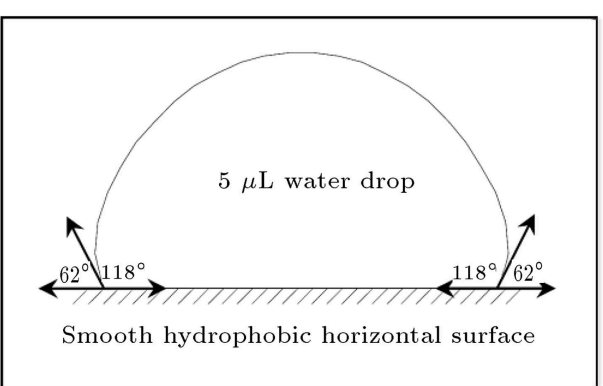

(b)

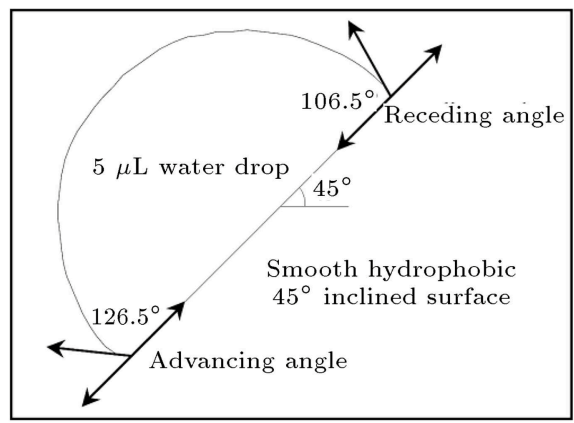

(d)

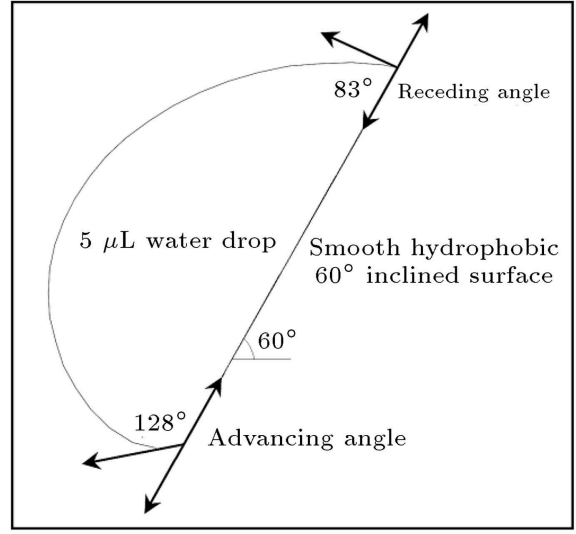

(f)

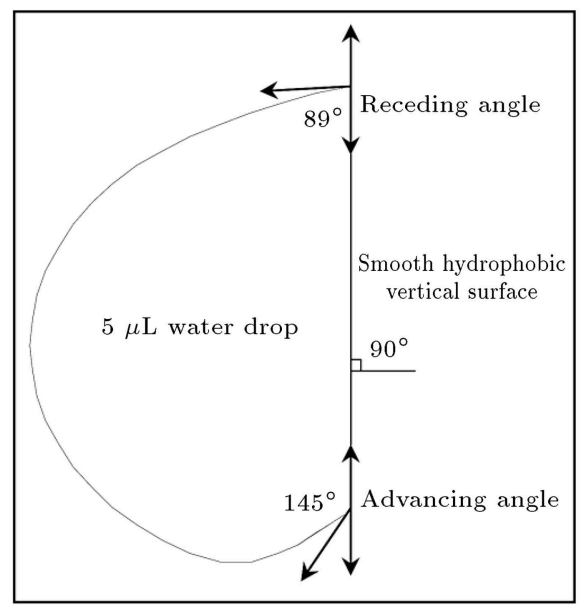

(h)

Figure 7. Experimental and numerical images of $5 \mu \mathrm{L}$ water drop on a smooth hydrophobic surface with different inclination angles. 
this figure. A slight difference between the contact angles measured at the right and the left of the drop, which is equal to $3^{\circ}$, is mainly due to the error in reading the angles as well as the variation of surface roughness. Of note, the mean value of these angles is used in the simulated program. Figure $7(\mathrm{~b})$ illustrates the predicted shape of the $5 \mu \mathrm{L}$ water drop on the same surface using the presented numerical technique. The predicted contact angle through the numerical simulation is $118^{\circ}$, which has a deviation of about $3 \%$ of the measured values.

Figure $7(\mathrm{c})$ represents the real shape of the $5 \mu \mathrm{L}$ water drop taken from the experimental measurements for the surface inclination angle of $45^{\circ}$. Figure $7(\mathrm{~d})$ shows the result of the presented numerical calculations for the same condition. A comparison of these two figures shows that the numerical calculations are in good agreement with the experimental measurements. Based on the measurements, advancing and receding contact angles of the droplet are $130.9^{\circ}$ and $109.8^{\circ}$, respectively. These two angles are $126.5^{\circ}$ and $106.5^{\circ}$ according to the numerical results. The error is $3.4 \%$ for the calculated advancing angle and $3 \%$ for the calculated receding angle.

The experimental and numerical results of the case of the smooth hydrophobic surface with a $60^{\circ}$ inclination angle are, respectively, shown in Figure $7(\mathrm{e})$ and (f). The advancing contact angles obtained through the tests and simulations are $131.6^{\circ}$ and $128^{\circ}$, respectively. In addition, the receding contact angles are $78.3^{\circ}$ and $83^{\circ}$ based on the experiments and numerical calculations. Percentage errors computed for the advancing and receding angles are $2.7 \%$ and $6 \%$, respectively.

Finally, the experimental and numerical results of the shape of $5 \mu \mathrm{L}$ water drop on a smooth hydrophobic vertical surface are, respectively, indicated in Figure $7(\mathrm{~g})$ and $(\mathrm{h})$. The measured advancing and receding contact angles are $139.6^{\circ}$ and $86.9^{\circ}$, respectively. In addition, the calculated advancing and receding angles are, respectively, $145^{\circ}$ and $89^{\circ}$. Percentage errors for the advancing and the receding angles are computed as $3.9 \%$ and $2.4 \%$, respectively. Of note, the percentage error is calculated by the following relation:

$$
\begin{aligned}
\operatorname{Error}(\%)= & \left(\frac{\mid \text { measured angle }- \text { calculated angle } \mid}{\text { measured angle }}\right) \\
& \times 100
\end{aligned}
$$

The presented results prove the validity of the numerical approach and computational code to predict the shape of a water droplet with any size on a smooth hydrophobic inclined surface. Therefore, the developed code was used to predict the shape and compute the advancing and receding contact angles of water droplets around the circular cylinders with various diameters.

Figure 8(a) illustrates the shape of sessile and pendant drops of $5 \mu \mathrm{L}$ volume around a circular cylinder of $25 \mathrm{~mm}$ diameter with the smooth hydrophobic surface. Figure $8(\mathrm{~b})$ and (c) show the predicted shape of water droplets with 10 and $15 \mu \mathrm{L}$ volumes around the same circular cylinder, respectively. Figure $8(d)$ illustrates the numerical predictions of the shape of $5 \mu \mathrm{L}$ water droplets around a cylinder of $35 \mathrm{~mm}$ diameter with the smooth hydrophobic surface. The shapes of 10 and $15 \mu \mathrm{L}$ water droplets around the same cylinder are illustrated in Figure 8(e) and (f), respectively. In addition, Figure $8(\mathrm{~g})$ indicates the present numerical simulations of the shape of $5 \mu \mathrm{L}$ water droplets around a cylinder of $70 \mathrm{~mm}$ diameter with the smooth hydrophobic surface. The shapes of 10 and $15 \mu \mathrm{L}$ water droplets around the same cylinder are depicted in Figure $8(\mathrm{~h})$ and (i). For each configuration in Figure 8, enlarged shapes of the five bottom droplets are also illustrated in the lower sections. For an equal drop size, the number of droplets increases as the cylinder diameter increases. Figure 8 also shows that cylinder diameter has minor effect on the shape of droplets, while the drop position has major consideration. Changing the size of drop also presented a slight variation of contact angles; however, their shape remains equivalent. However, Figure 8 illustrates that the drop shape and its contact angle on a specific surface is a function of both positions; in addition, drop size and heat transfer are affected by these parameters of the droplets.

In order to conduct a detailed analysis of the obtained results, the variations of both advancing and receding angles of 5,10 , and $15 \mu \mathrm{L}$ drops formed around cylinders of different diameters with respect to the drop circumferential position on the cylinders are plotted in Figure 9. In this figure, the horizontal axis includes the circumferential location of the drops around the cylinders. For example, number $-90^{\circ}$ on the lefthand side of the horizontal axis refers to the pendant drop formed at the bottom of the cylinder, while $+90^{\circ}$ on the right side refers to the sessile drop formed on the top. However, the values of the respective advancing and receding angles are included in the vertical axis.

According to Figure 9, the advancing and receding angles of the pendant and sessile drops (i.e., drops at the top and bottom of the cylinder) have the same values. In addition, the maximum value of advancing angle and the minimum value of receding angle are related to the drops formed on both sides of the cylinder on a horizontal line. Furthermore, the values of advancing and receding angles respectively decrease and increase when the cylinder diameter increases. In addition, Figure 9 shows that, generally, for a static 


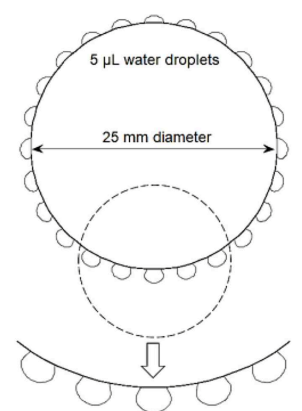

(a)

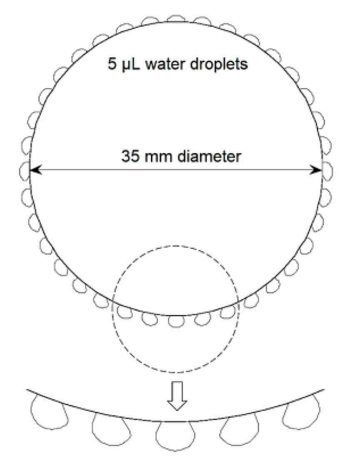

(d)

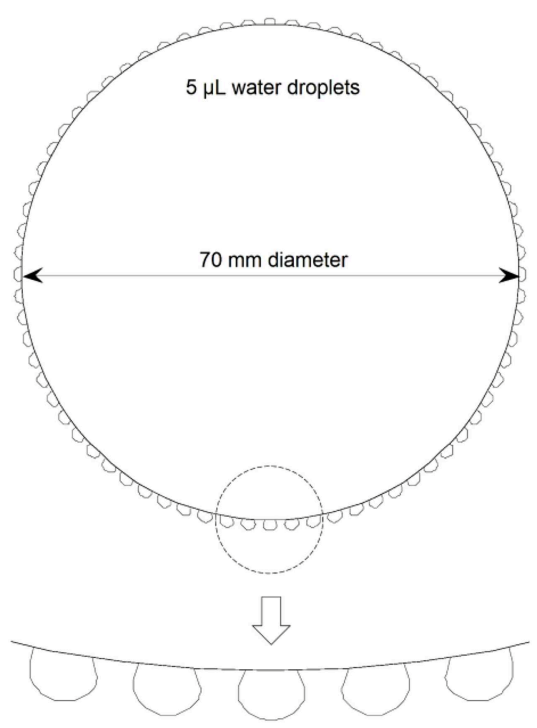

(g)

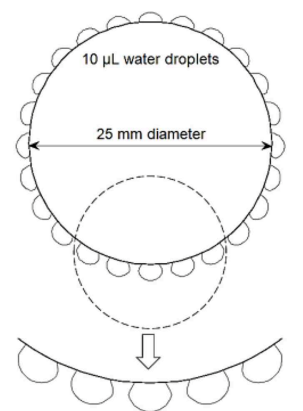

(b)

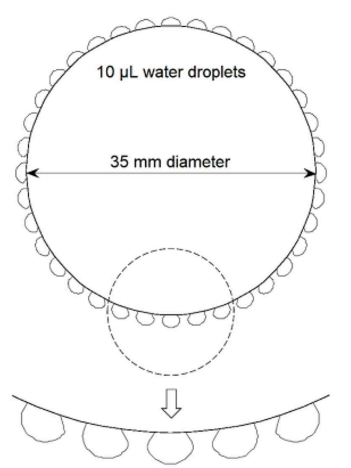

(e)

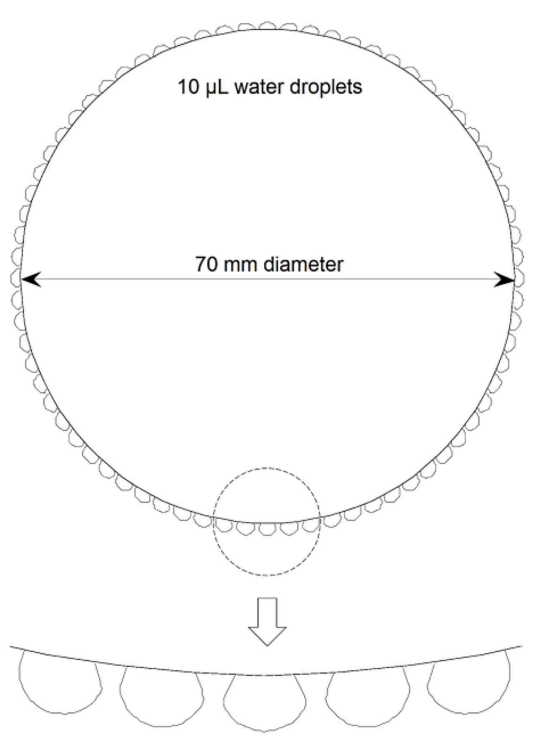

(h)

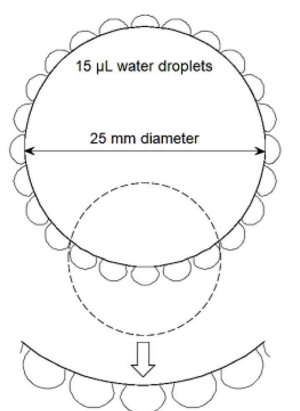

(c)

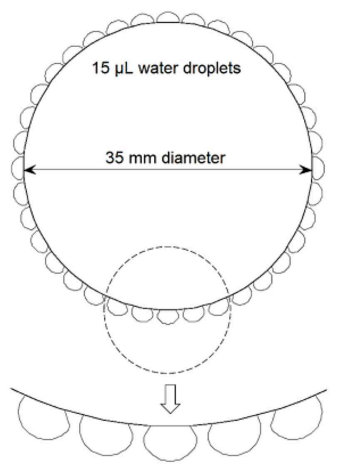

(f)

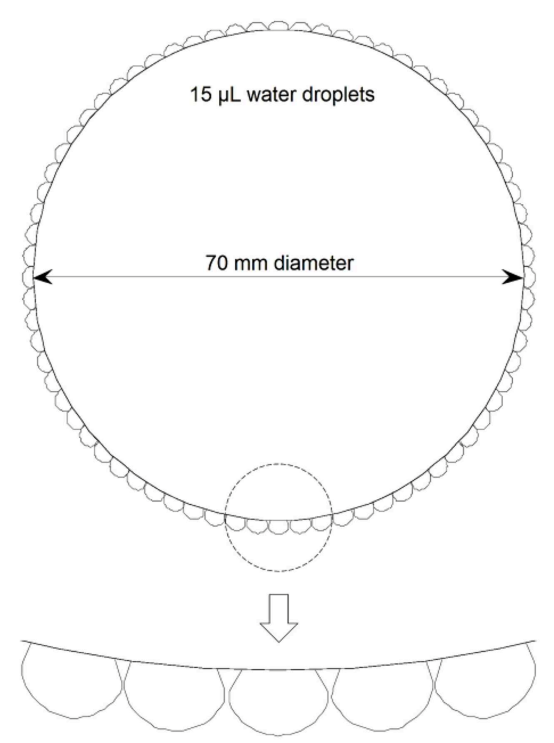

(i)

Figure 8. Numerically predicted shapes of 5,10 , and $15 \mu \mathrm{L}$ water droplets formed around horizontal cylinders with 25 , 35 , and $70 \mathrm{~mm}$ diameters.

water drop formed on a circular cylinder with a hydrophobic surface, advancing angle $\left(\psi_{a}\right)$ and receding angle $\left(\psi_{r}\right)$ are the functions of drop position $(\theta)$ as follows:

$$
\begin{aligned}
& \psi_{a}=A \theta^{2}+B \theta+C, \\
& \psi_{r}=A^{\prime} \theta^{2}+B^{\prime} \theta+C^{\prime} .
\end{aligned}
$$

In these equations, $\theta$ is in degrees and shows the location of the drop on the cylinder, as shown in Figure 10.

Moreover, coefficients in these two relations are functions of $d / D$, i.e., the ratio of drop diameter to cylinder diameter. Based on the calculations, an attempt is made to fit the contact angles in Figure 9 according to the shape of drop on various cylinders and various drop sizes. Accordingly, for Eqs. (6) and (7), these coefficients are determined by the least square 


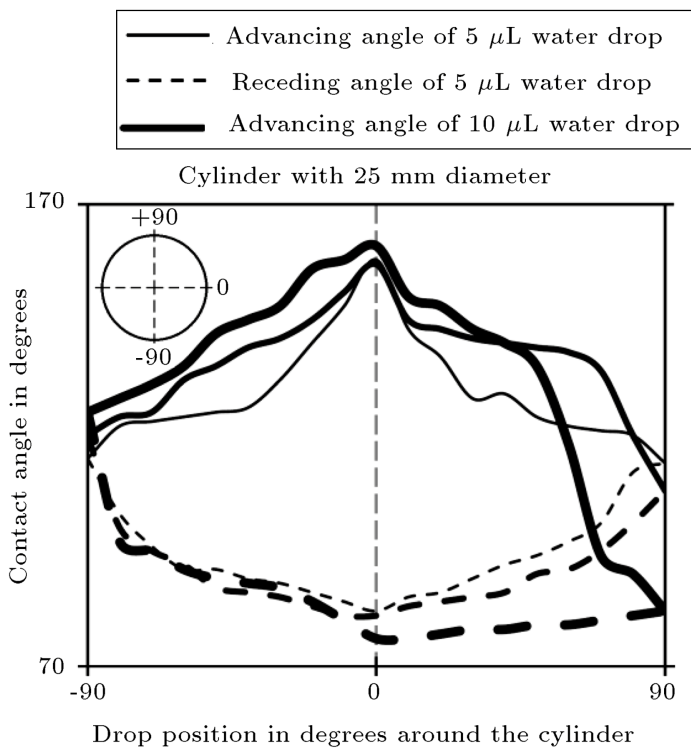

(a)
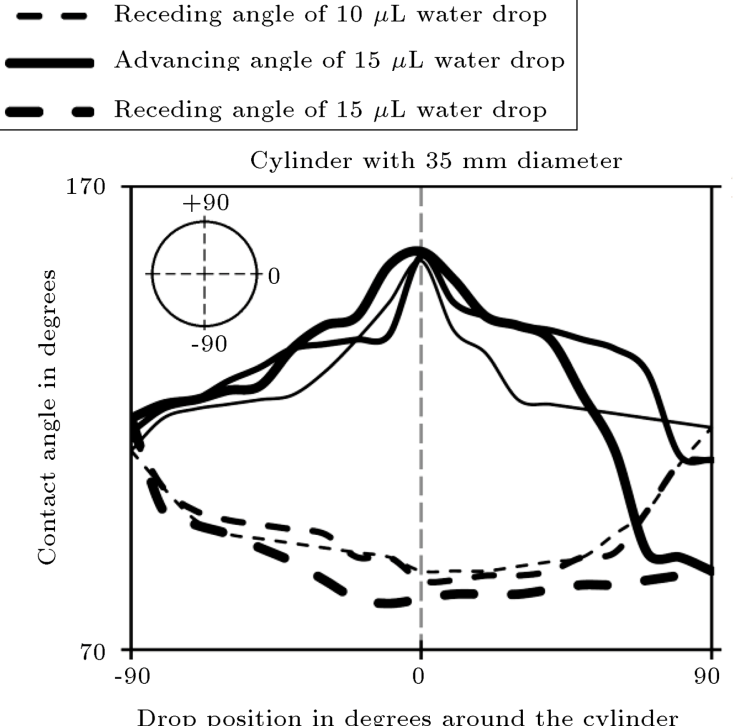

(b)

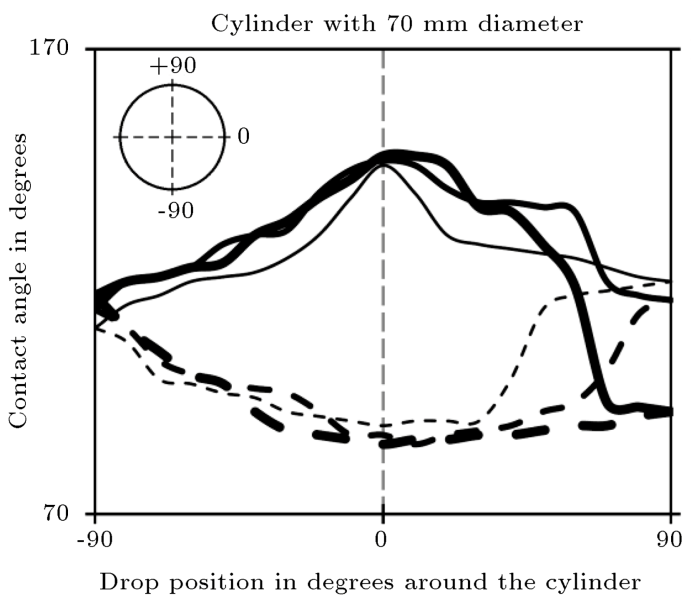

(c)

Figure 9. Values of advancing and receding angles for water droplets formed around a circular cylinder with diameters of (a) $25 \mathrm{~mm}$, (b) $35 \mathrm{~mm}$, and (c) $70 \mathrm{~mm}$.

method, and the values are provided in Eq. (8):

$$
\begin{aligned}
& A=-0.0226(d / D)-0.0022 \\
& B=-1.2423(d / D)+0.0683 \\
& C=131.9700(d / D)+130.4300 \\
& A^{\prime}=-0.0024(d / D)+0.0033 \\
& B^{\prime}=-0.9355(d / D)+0.0519 \\
& C^{\prime}=-92.3380(d / D)+92.7880
\end{aligned}
$$

Contact angle hysteresis is defined as the difference of advancing and receding angles. Figure 11 shows the variation of hysteresis values with respect to the drop circumferential position around the cylinders. For small drops, the values of hysteresis are small, in comparison with those of large drops. For all drop sizes, maximum hysteresis takes place when the drop is at $\theta=0^{\circ}$, and the horizontal line passes through the cylinder center. However, hysteresis becomes zero when the drop is at the top or at the bottom of cylinders, i.e., $\theta= \pm 90^{\circ}$. Hysteresis becomes more asymmetric as the drop size increases with respect to $\theta=0^{\circ}$. In addition, contact angle hysteresis decreases when the cylinder diameter increases.

\section{Conclusions}

Numerical simulations of sessile and pendant water droplets' shape with different sizes around circular cylinders with a smooth hydrophobic surface and 


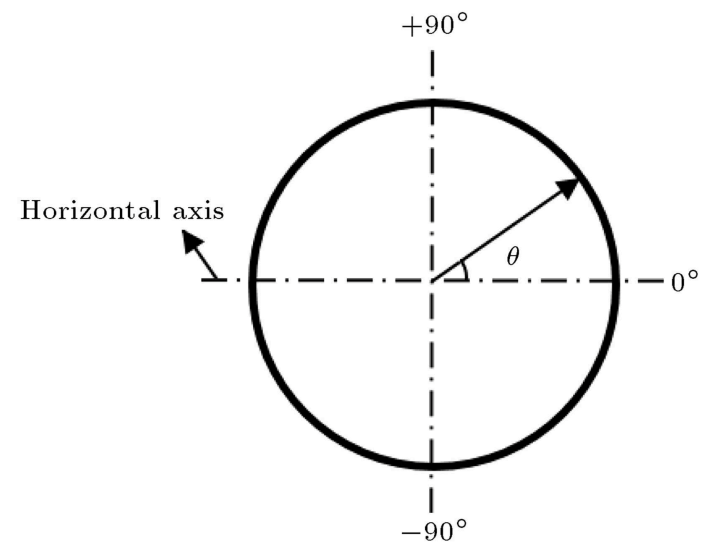

Figure 10. Location of a drop on the cylinder shown with angle.

various diameters are performed using the developed molecular dynamics algorithm. Based on the results, the following remarks can be concluded:

1. For all drop sizes and cylinder diameters, the curves of advancing and the receding angles get to each other at $\theta= \pm 90^{\circ}$. Note that $\theta=+90^{\circ}$ refers to the sessile drop formed at the top, and $\theta=-90^{\circ}$ refers to pendant drop formed at the bottom of the cylinder;
2. For all drop sizes, maximum of advancing angle curve happens almost at $\theta=0^{\circ}$, which refers to the drop formed near the horizontal line passing through the center of the cylinder. The minimum of the receding angle curve also happens at about this point. Thus, the maximum hysteresis takes place for a drop formed adjacent almost to the horizontal line;

3. The maximum percentage of variations at the advancing angle for the $5 \mu \mathrm{L}$ droplet formed around the cylinder is $36.3 \%$, while this quantity is equal to $71.3 \%$ for the case of the $15 \mu \mathrm{L}$ drop. For the maximum percentage of variations at the receding angle, the value of $35.6 \%$ is found for $5 \mu \mathrm{L}$ drop and $50 \%$ for $15 \mu \mathrm{L}$ one. Hence, the advancing and receding angles of small droplets exhibit almost equal variations. For large droplets, instead, the variation of the advancing angle is higher than that of the receding angle;

4. Both advancing and receding angles are subject to higher variations for large droplets than for small ones;

5. The contact angle for the sessile drop of $5 \mu \mathrm{L}$ formed at the top of the cylinder is slightly more than that of the pendant drop at the bottom.

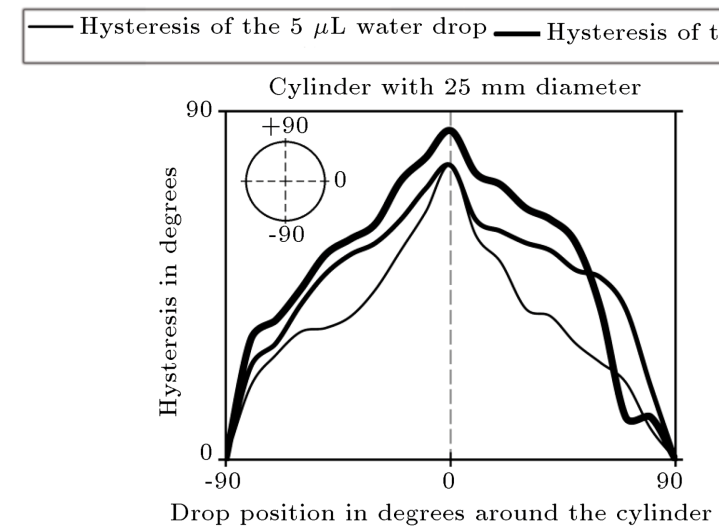

(a)

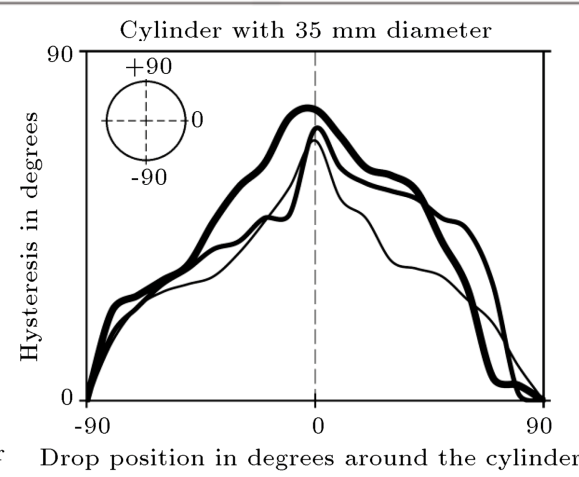

(b)

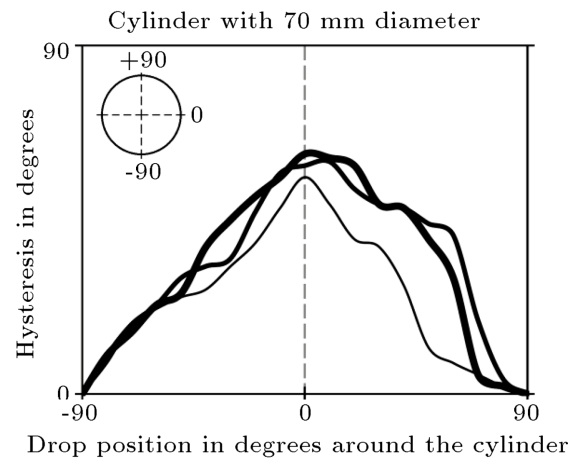

(c)

Figure 11. Values of hysteresis for water droplets formed around a circular cylinder with diameters of (a) $25 \mathrm{~mm}$, (b) 35 $\mathrm{mm}$, and (c) $70 \mathrm{~mm}$. 
However, the contact angle of the sessile drop of 10 $\mu \mathrm{L}$ formed on the top of the same cylinder is less than that of the pendant drop at the bottom. This difference is more evident for the droplet of $15 \mu \mathrm{L}$. Indeed, larger droplets have a greater tendency to slip over cylinder than smaller ones do;

6. The contact angles for various water drops on circular cylinders can be determined through the proposed correlations

\section{Acknowledgment}

The authors appreciate the partial support of "Iran's National Elites Foundation".

\section{References}

1. Rose, J. "Dropwise condensation theory and experiment: a review", Proc. Inst. Mech. Eng., Pt. A: J. Power Energy, 216(2), pp. 115-128 (2002).

2. Abu-Orabi, M. "Modeling of heat transfer in dropwise condensation", Int. J. Heat Mass Trans., 41(1), pp. 81-87 (1998).

3. Wu, Y.-T., Yang, C.-X., and Yuan, X.-G. "Drop distributions and numerical simulation of dropwise condensation heat transfer", Int. J. Heat Mass Trans., 44(23), pp. 4455-4464 (2001).

4. Vemuri, S. and Kim, K. "An experimental and theoretical study on the concept of dropwise condensation", Int. J. Heat Mass Trans., 49(3-4), pp. 649-657 (2006).

5. Rausch, M., Fröba, A., and Leipertz, A. "Dropwise condensation heat transfer on ion implanted aluminum surfaces", Int. J. Heat Mass Trans., 51(5-6), pp. 10611070 (2008).

6. Mei, M., Yu, B., Cai, J., and Luo, L. "A fractal analysis of dropwise condensation heat transfer", Int. J. Heat Mass Trans., 52(21-22), pp. 4823-4828 (2009).

7. Do Hong, S., Ha, M.Y., and Balachandar, S. "Static and dynamics contact angles of water droplet on a solid surface using molecular dynamics simulation", $J$. Colloid Interface Sci., 339(1), pp. 187-195 (2009).

8. Rausch, M., Leipertz, A., and Fröba, A. "Dropwise condensation of steam on ion implanted titanium surfaces", Int. J. Heat Mass Trans., 53(1-3), pp. 423$430(2010)$.

9. Lara, J.R. and Holtzapple, M.T. "Experimental investigation of dropwise condensation on hydrophobic heat exchangers part I: Dimpled-sheets", Desalination, 278(1-3), pp. 165-172 (2011).

10. Lara, J.R. and Holtzapple, M.T. "Experimental investigation of dropwise condensation on hydrophobic heat exchangers. Part II: Effect of coatings and surface geometry", Desalination, 280(1-3), pp. 363-369 (2011).

11. Mei, M., Yu, B., Zou, M., and Luo, L. "A numerical study on growth mechanism of dropwise condensation", Int. J. Heat Mass Trans., 54(9-10), pp. 20042013 (2011).
12. Guilizzoni, M. "Drop shape visualization and contact angle measurement on curved surfaces", J. Colloid Interface Sci., 364(1), pp. 230-236 (2011).

13. Park, J.-Y., Ha, M.-Y., Choi, H.-J., Hong, S.-D., and Yoon, H.-S. "A study on the contact angles of a water droplet on smooth and rough solid surfaces", J. Mech. Sci. Technol., 25(2), p. 323 (2011).

14. Sergi, D., Scocchi, G., and Ortona, A. "Molecular dynamics simulations of the contact angle between water droplets and graphite surfaces", Fluid Phase Equilib., 332, pp. 173-177 (2012).

15. Bonner III, R.W. "Correlation for dropwise condensation heat transfer: Water, organic fluids, and inclination", Int. J. Heat Mass Trans., 61, pp. 245-253 (2013).

16. Eral, H., 't Mannetje, D., and Oh, J. "Contact angle hysteresis: a review of fundamentals and applications", Colloid Polym. Sci., 291(2), pp. 247-260 (2013).

17. Santiso, E.E., Herdes, C., and Müller, E.A. "On the calculation of solid-fluid contact angles from molecular dynamics", Entropy, 15(9), pp. 3734-3745 (2013).

18. Wu, D., Wang, P., Wu, P., Yang, Q., Liu, F., Han, Y., $\mathrm{Xu}, \mathrm{F}$., and Wang, L. "Determination of contact angle of droplet on convex and concave spherical surfaces", Chem. Phys., 457, pp. 63-69 (2015).

19. Kim, J.-H., Kavehpour, H.P., and Rothstein, J.P. "Dynamic contact angle measurements on superhydrophobic surfaces", Phys. Fluids, 27(3), p. 032107 (2015).

20. Cheng, K., Naccarato, B., Kim, K.J., and Kumar, A. "Theoretical consideration of contact angle hysteresis using surface-energy-minimization methods", Int. J. Heat Mass Trans., 102, pp. 154-161 (2016).

21. Burt, R., Birkett, G., Salanne, M., and Zhao, X. "Molecular dynamics simulations of the influence of drop size and surface potential on the contact angle of ionic-liquid droplets", J. Phys. Chem. C, 120(28), pp. $15244-15250$ (2016).

22. Zhang, C., Liu, Z., and Deng, P. "Contact angle of soil minerals: A molecular dynamics study", Comput. Geo., 75, pp. 48-56 (2016).

23. Wu, S. and Ma, M. "A contact angle hysteresis model based on the fractal structure of contact line", $J$. Colloid Interface Sci., 505, pp. 995-1000 (2017).

24. Škvára, J., Škvor, J., and Nezbeda, I. "Evaluation of the contact angle from molecular simulations", Mol. Simul., 44(3), pp. 190-199 (2018).

25. Villa, F., Marengo, M., and De Coninck, J. "A new model to predict the influence of surface temperature on contact angle", Sci. Rep., 8, pp. 1-10 (2018).

26. Feng, X., Mo, Y., Zhao, Y., and Jiang, S. "Understanding the temperature and size dependence of the contact angle of $\mathrm{Cu} / \mathrm{Si}\left(\begin{array}{lll}1 & 1 & 1\end{array}\right)$ : A molecular dynamics study", Comput. Mater. Sci., 150, pp. 222-229 (2018).

27. Rosenholm, J.B., Peiponen, K.-E., and Gornov, E. "Materials cohesion and interaction forces", Adv. Colloid Interface Sci., 141(1-2), pp. 48-65 (2008). 
28. Javanmardi, M. and Jafarpur, K. "A molecular dynamics simulation for thermal conductivity evaluation of carbon nanotube-water nanofluids", J. Heat Trans., 135(4), pp. 042401-042401-9 (2013).

29. Wu, R., Liang, S., Pan, A., Yuan, Z., Tang, Y., Tan, X., Guan, D., and Yu, Y. "Fabrication of nano-structured super-hydrophobic film on aluminum by controllable immersing method", Appl. Surf. Sci., 258(16), pp. 5933-5937 (2012).

30. Minkowycz, W., Sparrow, E.M., and Murthy, J.Y., Handbook of Numerical Heat Transfer, 2nd Edn., John Wiley \& Sons, Inc. (2006).

31. Rapaport, D.C., The Art of Molecular Dynamics Simulation, 2nd Edn., Cambridge University Press (2004).

\section{Appendix}

The idea behind approximation of the cohesive force between two particles will be discussed here. Figure A.1 illustrates two adjacent particles, i.e., $i$ and $j$, in a drop. Each particle consists of a large number of molecules, and every molecule in either particle exerts forces on other molecules of both particles. In water, these forces are generally of two types: Lennard-Jones and Coulomb. Whatever type of force is considered, there is a finite range, out of which the effect of the force is negligible. Therefore, one can define a cutoff distance between a pair of molecules such that the interaction of them is calculated only when their distance is shorter than the cutoff distance. In conclusion, the cohesive force between two particles is due to the interaction of the molecules existing in an area close to their contact point. This area is shown by a small circle in Figure A.1. Contained in this circle are both a finite number of molecules belonging to particle $i$ and an equal number of molecules belonging to particle $j$. The cohesive force is, therefore, the result of the interactions that molecules in one half of this circle exert on the molecules in the other half.

A schematic diagram of a water molecule is shown in Figure A.2. Location of hydrogen and oxygen atoms along with the distance between the oxygen and each hydrogen atom is shown in this figure. Several models for the molecules of water can be identified in the literature, and the reader may refer to [30] to get more information about these models. Figure A.2 is plotted due to the values concerning the TIP4P model well introduced in $[30,31]$.

In this model, the positive electrical charges are assumed to be distributed equally over site $H$. The negative electrical charge, however, is considered to be located at site $M$ instead of site $O$. Sites $H$ and $M$ of a pair of molecules are responsible for exerting forces of Coulomb type, while Lennard-Jones interaction is considered to be originated from site $O$. As a result, the interaction between every pair of water molecules can be determined using the following relations:

$$
\begin{aligned}
& \vec{f}_{O_{i} O_{j}}=48\left(r_{O_{i} O_{j}}^{-14}-0.5 r_{O_{i} O_{j}}^{-8}\right) \vec{r}_{O_{i} O_{j}}, \\
& \vec{f}_{M_{i} M_{j}}=\left(\frac{4 b}{r_{M_{i} M_{j}}^{3}}\right) \vec{r}_{M_{i} M_{j}}, \\
& \vec{f}_{M_{i} H_{j}}=\left(-\frac{2 b}{r_{M_{i} H_{j}}^{3}}\right) \vec{r}_{M_{i} H_{j}}, \\
& \vec{f}_{H_{i} H_{j}}=\left(\frac{b}{r_{H_{i} H_{j}}^{3}}\right) \vec{r}_{H_{i} H_{j}} .
\end{aligned}
$$

In these relations, subscripts $i$ and $j$ refer to particles $i$ and $j$, respectively. Parameter $b$ is a constant equal to $183.5^{\circ}$ [31]. Moreover, $r_{O O}, r_{M M}, r_{M H}$, and $r_{H H}$ represent the distance between respective sites of both

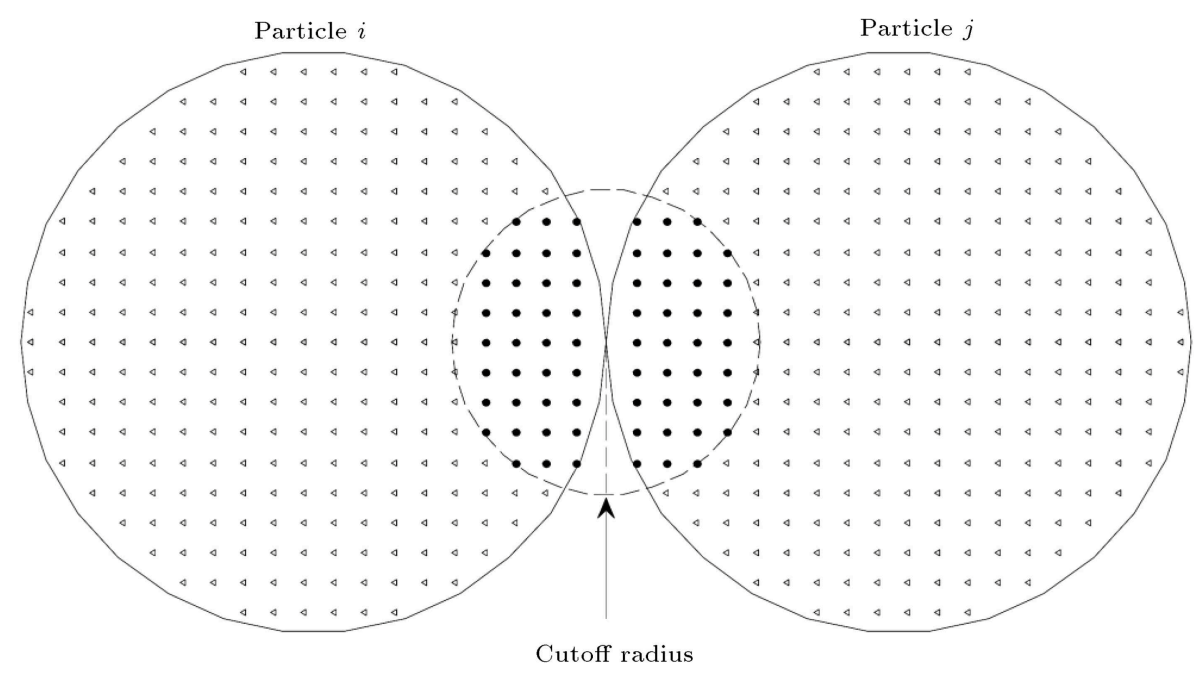

Figure A.1. Molecules of water contained in a pair of adjacent particles. 


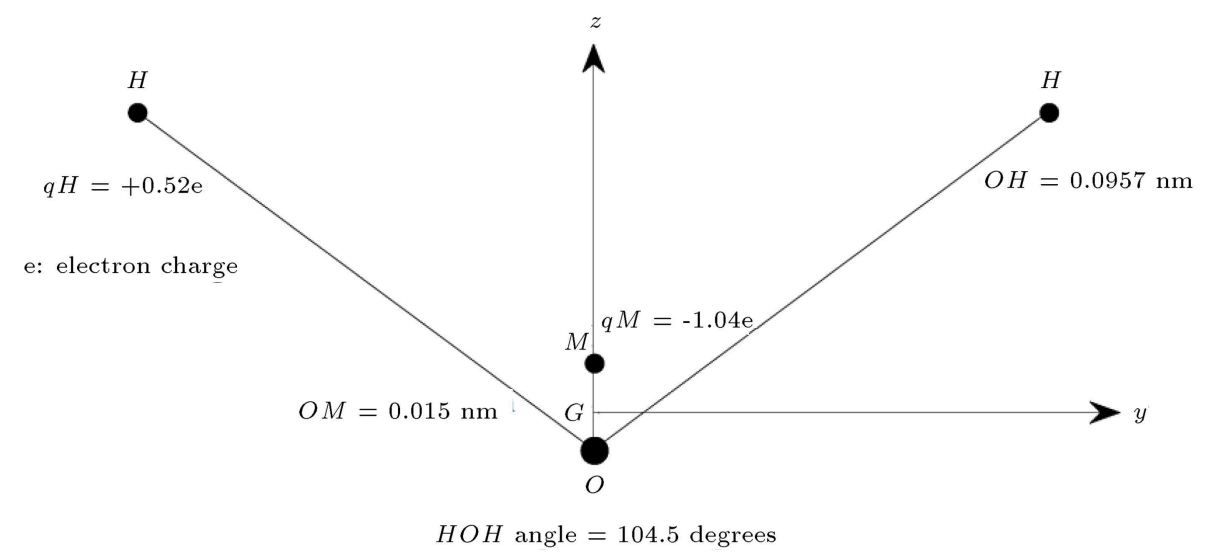

Figure A.2. Schematic of a water molecule in the TIP4P model.

molecules. In addition, $f_{O O}, f_{M M}, f_{M H}$, and $f_{H H}$ are the interactions between the respective sites of the molecules. It should be noted that the above relations are dimensionless. In order to return to the dimensional form of these relations, one should multiply the dimensionless distances by parameter $\sigma$ and the dimensionless forces by $\varepsilon / \sigma^{2}$. The values of $\sigma$ and $\varepsilon$ as reported in $[30,31]$ are $0.3154 \mathrm{~nm}$ and $1.0772 \times 10^{-21} \mathrm{~J}$, respectively. In conclusion, there are 10 interactions between these sites of every pair of molecules. The result of these interactions just returns the interaction between two molecules.

\section{Biographies}

Mohammad Javad Javanmardi obtained PhD of Mechanical Engineering from Shiraz University, Shiraz, Iran with a thesis entitled "Molecular dynamics simulation for nanofluids thermal conductivity evaluation". His field of research and interest is fluids heat transfer with the focus on numerical simulations. He has been appointed the Dean of Pasargad Higher Education Institute since 2017.

Kamran Hirbodi is a $\mathrm{PhD}$ Candidate in Mechanical
Engineering at Shiraz University, Shiraz, Iran. He worked in the field of phase change processes for his master's thesis and received the MSc degree at Shiraz University in September 2013. His research interests are experimental, numerical, and analytical modeling of heat and mass transfer and fluid flow, thermodynamics, solar energy, and biomechanics.

Abdullah Avara is a PhD Candidate in Mechanical Engineering at Shiraz University, Shiraz, Iran. He is working on dehumidification and convection heat transfer. He is a faculty member at the Mechanical Engineering Department of Persian Gulf University, Bushehr, Iran.

Mahmood Yaghoubi obtained the BSc and MSc degrees from Shiraz University, Shiraz, Iran and the PhD degree from Purdue University, Indiana, U.S.A, in 1978. He is a Professor of Mechanical Engineering at Shiraz University. $\mathrm{He}$ is also a Fellow of Iranian Academy of Sciences. His main research areas are convection heat transfer, system energy conversion, management and optimization, solar energy, and engineering education. 